\title{
Opportunities and Challenges in Delivering Remote Primary Care During the Coronavirus Outbreak
}

Vered Kaufman-Shriqui ( $\square$ veredks@ariel.ac.il )

Ariel University

\section{Michal Shani}

Tel Aviv University

Mona Boaz

Ariel University

Amnon Lahad

Hebrew University of Jerusalem

Shlomo Vinker

Tel Aviv University

Ruth Birk

Ariel University

\section{Research Article}

Keywords: Coronavirus, COVID-19, Health survey, Primary care, Telehealth, Telemedicine

Posted Date: January 17th, 2022

DOI: https://doi.org/10.21203/rs.3.rs-1163431/v1

License: (c) (i) This work is licensed under a Creative Commons Attribution 4.0 International License.

Read Full License 


\section{Abstract}

Background: Social distancing and lockdowns were implemented during the first period of the COVID-19 pandemic. Primary care physicians needed to adapt quickly to deliver remote care/telemedicine.

Methods: A cross-sectional, 47-item online Google Survey was distributed through the Israel Association of Family Physicians (IAFP) mailing list between March 31-May 5, 2020. The questionnaire included demographics, physician characteristics, and information on usage and perceived telemedicine quality. Sampling weights by sex and age groups were applied.

Results: 159 primary care physicians (10.6\% of registered IAFP members; $63.5 \%$ women; mean age $53.4 \pm 10.4$ years and median professional experience 21.3 years) replied to the survey. The majority $(59.7 \%)$ of the participants performed a mixture of in-person along with phone counseling. About $40 \%$ had no former telemedicine experience. The majority indicated that telephone and video formats were inferior to in-person consultation ( $68 \%, 57.1 \%$ online and phone, respectively). The overall counseling quality grade (on a 1-10 scale, ) median (IQR)) was 6.2 (3) for telephone and 7(2) for video. While $66.9 \%$ reported experiencing no challenges, $10 \%$ had technical problems, $10 \%$ interpersonal problems, $5.6 \%$ scheduling difficulties, and $7.5 \%$ other difficulties. Majority of $56.6 \%$ physicians indicated they prescribed more antibiotics, $16.4 \%$ sent more blood tests, $24.5 \%$ referred more to experts, and $49.7 \%$ referred more to imaging in comparison to usual counseling. Higher phone quality score was significantly associated with physicians who indicated not prescribing more antibiotics during the pandemic $(\mathrm{OR}=0.30,95 \% \mathrm{Cl} 0.134$ $0.688, p=0.004)$.

Higher online quality score was associated with physicians who indicated not sending more blood tests during the pandemic $(\mathrm{OR}=0.0695 \% \mathrm{Cl} 0.008-0.378, \mathrm{P}=0.003)$.

Conclusions: Our findings suggest telehealth holds considerable promise for counseling in the primary care setting. However, interpersonal challenges raised by physicians should be understood in-depth to develop tailored training and further examine it in randomized trials while integrating patient-reported outcomes. Finally, further research on utility, cost, and cost-efficiency during remote counseling with follow-ups, medical prescribing, and additional referrals is needed.

\section{Introduction}

Primary care is characterized by long-term patient-physician relationships and continuity. The introduction of telemedicine and the ability to communicate without in-person clinic visits has created new modalities for interaction with patients. Telemedicine has been found to be effective and to improve clinical outcomes in a variety of medical conditions, including wound care, psychiatry, and chronic conditions like diabetes mellitus and hypertension (1). Informational videos providing instruction in selfmonitoring and health education and awareness tailored to specific morbidities have been shown to reduce hospitalizations among people at high risk for re-hospitalization with heart failure, chronic obstructive pulmonary disease (COPD), diabetes mellitus, or hypertension (2). Additionally, at a large 
hospital outpatient setting staffed by physicians trained to deliver telemedicine, both patients and clinicians found no difference in the quality of virtual vs. in-person office visits (3).

Video consultations were rated as positive experiences, especially among working people or patients with mobility problems (4). In a system allowing patient access to medical care $24 \mathrm{~h}$ a day via web and phoneenabled virtual visits, $85 \%$ of patients rated their satisfaction with their telemedicine physician at the highest rank(5). High satisfaction rates were also obtained by patients with celiac who used telemedicine during the COVID-19 pandemic (6). However, according to a national survey, fewer than $10 \%$ of family physicians in the USA provided e-visits, despite patient satisfaction (7). Common barriers to telemedicine use included technical challenges, resistance to change, as well as patient age and level of education (8). Physicians' important concerns working in a pediatric telemedicine service were difficulties diagnosing from a distance and treating unfamiliar patients (9). A recent Cochrane review identified gaps in knowledge of physicians' perspective, experiences, adherence, and satisfaction in delivering medical care using mobile technologies (10).

The COVID-19 pandemic has forced rapid adjustment for family physicians to practice telemedicine. The general lockdown coupled with patient anxiety about clinic visits has forced family physicians to adopt telemedicine and distance communication with patients instead of in-person encounters. A recent study conducted among primary care physicians in Israel indicated that daily use of WhatsApp in professional practice reduced the need for in-person counseling (11).

In the current study, we aimed to evaluate physician experiences and attitudes to the change in practice paradigm during the COVID-19 pandemic.

\section{Methods}

\section{Overall Study Design and Plan}

A convenience sample of primary care physicians replied to an online survey distributed via e-mail by the Israeli Association of Family Physicians to all members. The study used a Google Survey platform. Data collection was performed from March 31-May 5, a period of time during which the Israeli population was under wide-scale lockdown.

\section{Ethics}

The study was approved by the Institutional Ethics Committee of Ariel University. Each participant provided electronic written informed consent prior to responding to the survey.

\section{Survey Tool}


The survey included 47 questions, including sociodemographic information (6 items), characteristics of routine primary care setting and employment (8 items), characteristics of primary care setting during the outbreak (4 items), features experiences and quality of telemedicine including phone or video or a combination of them (8 questions on phone counseling and additional 12 questions on video counseling), physician experiences ( 5 items), and perceived patient experiences ( 4 items). Some questions were modified from a telemedicine questionnaire for healthcare workers $(12,13)$. The questions selected requested information on overall quality, technical quality, clinical quality, and organizational difficulties (on a scale of 1-10) and additional questions on technical difficulties and future use (on a scale of 1-4). Survey participants were also asked to indicate their work setting, the number of registered patients, the weekly number of work hours, if and how they continued to work during the COVID-19 outbreak, and the proportion of patients with whom they corresponded using e-mail, WhatsApp, or phone prior to the outbreak. Additionally, physicians were asked whether non-face-to-face meetings were an option and to estimate the percentage of their patients who were invited to the clinic following a phone or video consultation (five categories: never, $25 \%, 50 \%, 75 \%$, all). Respondents were asked to rate the quality of remote platforms to in-person counseling. Participants were requested to describe the challenges that they experienced during counseling sessions. This information was thematically analyzed by two researchers who reached a consensus on the main topics. Additionally, participants used a 10-point Likert scale to estimate client comfort level during remote counseling. Participants replied to the survey in Hebrew. Prior to publishing the survey online, the survey questions and usability of the online platform were referred to a focus group of 10 potential participants, who were asked to assess readability and clarity. Based on their feedback, minor amendments were made. The final questionnaire was reviewed by ten expert primary care physicians providing expert validity.

\section{Comparison between the study population and the general population of primary care professionals in Israel}

To determine the representativeness of the study sample, participant age by sex distributions were compared to those of the target population (Israeli primary care physicians), using the most recent data available (14). As shown in Table 1, the survey population was younger and included a greater proportion of women than the target population. Age distribution differed significantly by sex, such that the study sample included a greater proportion of both men and women between the ages $41-60$, while the target population was characterized by a greater proportion of men between the ages of 51-70 years, and women between the ages of 41-70 years $\left(x^{2}=225.29, D F=5, p<0.001\right)$. Sample weights were created based on the national age and sex distribution and applied to the survey population. 
Table 1

Comparison between the distribution of family physicians by sex and age group in the survey and the country*

\begin{tabular}{|c|c|c|c|c|}
\hline $\begin{array}{l}\text { Age } \\
\text { group }\end{array}$ & $\begin{array}{l}\text { Men in the } \\
\text { survey } n,(\%)\end{array}$ & $\begin{array}{l}\text { Men in the profession in } \\
\text { the country }(n, \%)\end{array}$ & $\begin{array}{l}\text { Women in the } \\
\text { survey }(n, \%)\end{array}$ & $\begin{array}{l}\text { Women in the profession } \\
\text { in the country }(n, \%)\end{array}$ \\
\hline $\begin{array}{l}\text { Lower } \\
\text { than } 30\end{array}$ & $0(0)$ & $75(2.7)$ & $1(1.0)$ & $27(1.2)$ \\
\hline $31-40$ & $8(14.0)$ & 336 (12.0) & $22(21.8)$ & $297(13.2)$ \\
\hline $41-50$ & $20(35.1)$ & $541(19.5)$ & 39 (38.6) & $573(25.5)$ \\
\hline $51-60$ & $14(24.6)$ & $809(29.1)$ & $29(28.7)$ & 746 (33.2) \\
\hline $61-70$ & $10(17.5)$ & 870 (31.3) & $10(9.9)$ & 527 (23.5) \\
\hline $\begin{array}{l}71 \text { and } \\
\text { above }\end{array}$ & $5(8.8)$ & $130(4.7)$ & $0(0)$ & $59(2.6)$ \\
\hline Missing & & $20(0.7)$ & & $15(0.7)$ \\
\hline Total & 57 & 2781 & 101 & 2244 \\
\hline
\end{tabular}

\section{Data Analysis}

SPSS v. 25.0 (IBM Inc., USA) was used for all statistical analyses. First, the weighted characteristics of study population were described., Distributions of continuous variables were assessed for normality using the Kolmogorov-Smirnov test. Normally distributed continuous variables are described using mean \pm standard deviation. Continuous data with distributions significantly deviating from normal are described as median (interquartile range). Categorical variables such as the percentage of participants providing a given response were described using frequency counts and expressed as $n$ (\%). Associations between sociodemographic and occupational characteristics of physicians and the overall quality of phone and online counseling were examined using survey-weighted multiple logistic regression analyses with stepwise variable selection. The independent variables associated with the overall quality variable at a significance level of $p \leq 0.10$ were considered for inclusion in multivariate models. Two separate models were developed, and the median value of the quality score at each type of consultation was assigned as the cutoff value. All tests are two-sided and considered significant at $p<0.05$.

\section{Results}

\section{The survey participants}


Of 1,500 registered members of the Israel Association of Family Physicians (IAFP), 159 replied to the survey (10.6\%). Table 2 describes the characteristics of survey participants. The mean participant age was $53.4 \pm 10.4$ years and most were female. The majority (90\%) of the participants were specialists in family medicine, and $60 \%$ were employees of one of the national health maintenance organizations (HMO), while $30.8 \%$ were independent physicians, and $8.8 \%$ worked in both employment settings. Median professional experience was 20.0 years. The majority of physicians $(59.8 \%)$ reported more than 1,000 patients in their clinics. Most participants (73.6\%) indicated that during routine times, $50 \%$ of their patients or more would require a physical examination. A greater proportion of the physicians indicated that the most prevalent communication type with patients prior to the Covid-19 outbreak was e-mail correspondences, followed by phone calls and, to a lesser extent, WhatsApp messages.

\section{Table 2. Characteristics of Survey Participants}


Characteristic

Sex $n$, (\% female)
Age, years (Mean $\pm S D)$

Result

$n=159$

$53.4 \pm 10.4$

$101.0(63.5)$

Weighted:

$49.9 \%$

Specialization in medicine $n$ (\%)

Family medicine

Intern in family medicine

$9.0(5.7)$

Practicing family medicine with no specialization

$4.0(2.5)$

Other medical specialization

$3.0(1.9)$

Years of professional experience Median (IQR)

$21.3(17.0)$

The main form of employment $n$ (\%)

Employee $96.0(60.4)$

Independent physician $49.0(30.8)$

Both employee and independent physician $14.0(8.8)$

How many registered patients do you have? $n(\%)$

More than 1500

$45.0(28.3)$

Between 1000-1500

$50.0(31.5)$

Between 500-1000 $46.0(28.9)$

Less than 500

Number of hours/week performing counseling (prior to the COVID-19 outbreak) $($ Mean \pm SD)

At routine times, which proportion of your patients' visits require a physical examination $n(\%)$

All patients

$75 \%$ of the patients $24.0(15.1)$

$50 \%$ of the patients $89.0(56.0)$

$25 \%$ of the patients or less $42.0(26.4)$

Before the Corona crisis, did you give your cell phone number to patients routinely? $n(\%)$ 
Before the Corona crisis, did you routinely hold WhatsApp correspondence with patients? n(\%)

Yes

Before the Corona crisis, did you routinely communicate with patients via your e mail? $n(\%)$

Yes

Abbreviations: IQR, interquartile range

\section{Characteristics of primary care counseling during COVID-19 outbreak}

Table 3 describes the characteristics of primary care counseling during the study period. The majority of physicians reported full-time employment (92.5\%) but reported a decrease in meeting patients to $18.3 \pm 12.3$ hours on average compared to $27.9 \pm 10.7$ mean hours prior to the pandemic. While $41.5 \%$ reported no change in client characteristics, $40.3 \%$ reported accepting younger clients, and $9.4 \%$ older clients. 
Table 3

Characteristics of counseling since the COVID-19 outbreak $^{\mathrm{a}}$

\section{Characteristic}

Result

$\mathrm{N}=159$

Work capacity after the onset of the COVID-19 n (\%)

Full-time

Part-time

Number of hours/week performing in-patient counseling (since the COVID-19 outbreak)

$18.3 \pm 12.3$ $($ Mean \pm SD)

Changes in patients characteristics during the COVID-19 outbreak

No changes in patients characteristics

Younger patients

Older patients

Other

Was it possible for the patients to schedule a non-face-to-face meeting in your clinic?

yes $n,(\%)$

The setting of counseling after the COVID-19 outbreak

Usual counseling and phone counseling

Only phone counseling

Only online counseling

Only phone and online counseling

Usual counseling as well as phone and online counseling

$52(32.7)$

${ }^{a}$ Calculated with the application of sample weights.

During the pandemic, the majority of the physicians (92.5\%) employed some form of telemedicine. Types of mixed counseling were frequent, with the majority of the participants who performed a mixture of usual face-to-face counseling with phone counseling (59.7\%). The greatest percentage of respondents who performed online counseling used a mixture of video platforms. The most frequently used platform was WhatsApp (53.9\%), with $22.2 \%$ who used it as the only option, followed by ZOOM (22.2\%) and Microsoft Teams (16.6\%). An additional 7.3\% used Facebook Messenger, Skype, and Unicko.

Characteristics of primary care consultation over the phone and when using online video platforms are presented in Table 4. 
Table 4

Characteristics of quality of telemedicine using the phone and online platforms ${ }^{*}$

Question/Response

Result

Counseling over the phone $\mathrm{n}(\%)$

$N=157$

Previous experience with performing telephone counseling

Yes

Limited experience

No

How would you compare the duration of phone counseling in comparison to face-to-face counseling?

Similar in duration to face-to-face counseling

Much longer than face-to-face counseling

Slightly longer face-to-face counseling

Much shorter than face-to-face counseling

Slightly shorter than face-to-face counseling

Percentage of the patients that were requested to arrive at a face-to-face meeting following a telephone counseling

None

$25 \%$

$50 \%$ and above

Percent of patients who shared photographs or videos during the meeting [median

(interquartile range) $]^{\mathrm{a}}$

* Calculated with the application of sample weights.

a Open scale, replies were numerically restricted to 0-100 percent.

b Items scored on a 10-point Likert scale ranging from 1-10, where 1= "very low" to 10 "very high."

${ }^{c}$ Other difficulties included themes of trust, age difficulties, hearing and sight disability which prevented patients from communicating well using the suggested telemedicine, and a desire to see the physician in person. 


\section{Question/Response}

Result

How would you compare phone counseling to usual counseling (face-to-face)? n (\%)

Superior to face-to-face counseling

Similar to face-to-face counseling

Inferior to face-to-face counseling

Not certain

The overall quality of counseling [median (interquartile range)] ${ }^{\mathrm{b}}$

Level of physician's convenience in performing phone counseling $n,(\%)$

Highly convenience

Reasonably convenience

Not highly convenient

Not convenient at all

Intention to use telephone counseling in the future $\mathrm{n}(\%)$

Yes

Yes, while combining phone and face-to-face counseling

Not certain

No

\section{Counseling using an online (video) platform}

Previous experience with performing online video counseling $n(\%)$

Yes

* Calculated with the application of sample weights.

a Open scale, replies were numerically restricted to 0-100 percent.

b Items scored on a 10-point Likert scale ranging from 1-10, where 1= "very low" to 10 "very high."

${ }^{c}$ Other difficulties included themes of trust, age difficulties, hearing and sight disability which prevented patients from communicating well using the suggested telemedicine, and a desire to see the physician in person. 
How would you compare the duration of phone counseling in comparison to face-to-face counseling? $n(\%)$

Similar in duration to face-to-face counseling

Much longer than face-to-face counseling

Slightly longer face-to-face counseling

Much shorter than face-to-face counseling

Slightly shorter than face-to-face counseling

Percentage of the patients that were requested to arrive at a face-to-face meeting following a telephone counseling

$\mathrm{n}(\%)$

None

$25 \%$

$50 \%$ and above

How would you compare online counseling to usual counseling (face-to-face)? n (\%)

Superior to face-to-face counseling

Similar to face-to-face counseling

Inferior to face-to-face counseling

Not certain

* Calculated with the application of sample weights.

a Open scale, replies were numerically restricted to 0-100 percent.

b Items scored on a 10-point Likert scale ranging from 1-10, where 1= "very low" to 10 "very high."

${ }^{c}$ Other difficulties included themes of trust, age difficulties, hearing and sight disability which prevented patients from communicating well using the suggested telemedicine, and a desire to see the physician in person. 


\section{Question/Response}

Result

Level of physician's convenience in performing video counseling $n,(\%)$

Highly convenience

Reasonably convenience

Not highly convenient

Not convenient at all

Intention to use telephone counseling in the future $\mathrm{n}(\%)$

Yes

Yes, while combining phone and face-to-face counseling

Not certain

No

Quality of counseling [median (interquartile range)]*

Overall quality ${ }^{b}$

Technical quality ${ }^{b}$

Physician challenges in performing the online counseling

Organizational difficulties ${ }^{b}$

Technical difficulties, $\mathrm{n}(\%)$

Difficulties due to lack of physical examination, $n(\%)$

Scheduling difficulties, $\mathrm{n}(\%)$

* Calculated with the application of sample weights.

a Open scale, replies were numerically restricted to $0-100$ percent.

b Items scored on a 10-point Likert scale ranging from 1-10, where 1= "very low" to 10 "very high."

${ }^{c}$ Other difficulties included themes of trust, age difficulties, hearing and sight disability which prevented patients from communicating well using the suggested telemedicine, and a desire to see the physician in person. 
Other $^{\mathrm{c}}, \mathrm{n}(\%)$

In comparison to the face-to-face meeting, have you prescribed more antibiotics? n (\%) N N=159

Yes

Prescribed as usual

In comparison to the face-to-face meeting, have you sent more blood tests? $\mathrm{n}(\%)$

Yes

Blood tests as usual

In comparison to the face-to-face meeting, have you referred patients to experts more than usual? $\mathrm{n}(\%)$

Yes

Referrals as usual

In comparison to the face-to-face meeting, have you referred to more imaging tests? $\mathrm{n}(\%)$

Yes

Imagining as usual

Patient Outcomes

Perceived level of patient comfort in receiving remote counseling (phone or video) [median (interquartile range])

Types of difficulties reported by patients during online (video) or phone counseling $n(\%)$

Technical difficulties

* Calculated with the application of sample weights.

a Open scale, replies were numerically restricted to 0-100 percent.

b Items scored on a 10-point Likert scale ranging from 1-10, where 1= "very low" to 10 "very high."

${ }^{c}$ Other difficulties included themes of trust, age difficulties, hearing and sight disability which prevented patients from communicating well using the suggested telemedicine, and a desire to see the physician in person. 
* Calculated with the application of sample weights.

a Open scale, replies were numerically restricted to 0-100 percent.

b Items scored on a 10-point Likert scale ranging from 1-10, where $1=$ "very low" to 10 "very high."

${ }^{c}$ Other difficulties included themes of trust, age difficulties, hearing and sight disability which prevented patients from communicating well using the suggested telemedicine, and a desire to see the physician in person.

\section{Consultation over the phone}

More than one-third of respondents (35.7\%) indicated they had no previous experience performing consultation over the phone prior to the COVID-19 pandemic, while another third (32.5\%) had previous experience. The majority (58.6\%) of respondents indicated phone counseling was of shorter duration than in-person visits. Of these, $14.6 \%$ indicated that consultation was significantly shorter than in-person consultations. Most respondents (89.9\%) indicated that following phone counseling, they needed to invite about $25 \%$ of the patients to the clinic for an in-person visit. Only 4\% (5 IQR) indicated that patients shared videos or photos with them during phone counseling. The majority of respondents $(68.8 \%)$ indicated that phone consultation was inferior to in-person visits. On a scale of 1-10, the median (IQR) rate for the overall quality of phone counseling was 6.2 (3.0). Many respondents (62.1\%) thought phone counseling was reasonably or highly convenient. The majority $(80.4 \%)$ of respondents indicated they intend to use phone counseling in the future and only $5 \%$ indicated they would not use it.

Consultation via online (video) platforms

Many (41.1\%) of respondents indicated they had no previous experience performing consultation using online platforms prior to the COVID-19 pandemic, while 39.3\% reported having had previous experience. The majority (64.1\%) of respondents indicated online counseling was either similar in length or of shorter duration than in-person visits. Most respondents (75.0\%) indicated that following online counseling, they needed to invite about $25 \%$ of the patients to the clinic. The majority of respondents $(57.1 \%)$ indicated online consultation was inferior to in-person visits. On the other hand, most respondents $(73.2 \%)$ thought online counseling was reasonably or highly convenient, and only $1.8 \%$ thought it was not convenient. The majority $(80.3 \%)$ of respondents indicated they intend to use online counseling in the future. 


\section{Challenges and qualities of remote counseling}

On a scale of 1-10, the median (IQR) rate for the overall quality of online counseling was 7 (2), the technical quality 6(2), and for organizational difficulties, 7(3). Physicians reported a variety of challenges in performing online consultations. While $66.9 \%$ reported experiencing no challenges, $10 \%$ had technical problems, $10 \%$ interpersonal problems, $5.6 \%$ scheduling difficulties, and $7.5 \%$ other difficulties. In reply to questions addressing characteristics of the medical treatment, in any remote platform (either phone or online), $56.1 \%$ indicated they prescribed more antibiotics, $16.4 \%$ sent more patients than usual to perform blood tests, $24.5 \%$ indicated an increase in referral to expert physicians and $49.7 \%$ indicated an increase in referral to imaging. Main themes which emerged from an open-ended question that requested further information on challenges identified four populations with which telemedicine was challenging in particular: older people; low socioeconomic status populations; people with hearing disability; and people with low technological literacy.

In reply to the question, "What are your primary needs to perform a higher quality remote?" the majority of physicians described a need for high-quality equipment (camera, headphones, adequate chair for long sitting); infrastructure (strong internet network resources, adequate light); and a better scheduling system for the patients with reminders to prevent missed visits. Additionally, due to the situation of working under lockdown when some physicians were also parents for young children, physicians highlighted the need for assistance with childcare while they are at work.

\section{Patient Outcomes}

Physician-reported patient outcomes included the patient's level of comfort and difficulties as perceived by the participating physician. The median (IQR) level of patient comfort was $8(3)$. Physicians reported that $46.3 \%$ of their patients reported no difficulties with telemedicine methods, but the rest reported at least one problem. The most frequently reported patient problems were technical difficulties $(30.0 \%)$, interpersonal challenges (20.6\%), lack of physical checkup and measurements (13.8\%), problems due to holding the meeting in the patient's home environment (4.4\%), and $8.8 \%$ who reported on other difficulties.

Associations between sociodemographic and occupational characteristics of primary care physicians and the overall quality of phone and online counseling

In a multivariate logistic regression in which the dependent variable was the total quality score of counseling using the phone greater than 6 , the only significant predictor variable was prescribing more antibiotics. Specifically, respondents who reported prescribing more antibiotics had $3.03 \%$ lower odds for a higher quality score $(\mathrm{OR}=0.30395 \% \mathrm{Cl} 0.134-0.688, \mathrm{p}=0.004)$ (Table $5 \mathrm{a})$. In a multivariate logistic regression in which the dependent variable was the total quality score of counseling using an online video platform higher than a median value of 7 , which included sex, age, and previous experience in video counseling, an increase in referrals to blood tests was the only significantly associated variable with a $6 \%$ reduction in odds of reporting a greater quality score $(\mathrm{OR}=0.0695 \% \mathrm{Cl} 0.008-0.378, \mathrm{P}=0.003)$ (Table $5 \mathrm{~b})$. 
Table 5

a Factors associated with a higher overall quality score of phone counseling in a multivariable logistic regression analysis*a

\begin{tabular}{|c|c|c|c|}
\hline & $\begin{array}{l}\text { Odds } \\
\text { Ratios }\end{array}$ & $95 \% \mathrm{Cl}$ & $\begin{array}{l}\text { p- } \\
\text { value }\end{array}$ \\
\hline Age (years) & 0.99 & $\begin{array}{l}0.959- \\
1.038\end{array}$ & 0.91 \\
\hline Sex (men vs. women) & 1.02 & $\begin{array}{l}0.468- \\
2.204\end{array}$ & 0.968 \\
\hline Prescribing more antibiotics & 0.30 & $\begin{array}{l}0.134- \\
0.688\end{array}$ & 0.004 \\
\hline $\begin{array}{l}\text { Previous experience using a phone in primary care counseling (no } \\
\text { experience vs. experience) }\end{array}$ & 1.14 & $\begin{array}{l}0.499- \\
2.607\end{array}$ & 0.756 \\
\hline Constant & 5.34 & & 0.145 \\
\hline \multicolumn{4}{|l|}{ a Calculated with the application of sample weights. } \\
\hline \multicolumn{4}{|c|}{$\begin{array}{l}\text { * Quality score was calculated as the sum of scores of the item questions on quality, technical quality, } \\
\text { clinical quality, and organizational difficulties (on a scale of 1-10). Higher quality score was assigned } \\
\text { a total quality score higher than the median value of } 6 \text { points for performing telemedicine using the } \\
\text { phone. }\end{array}$} \\
\hline
\end{tabular}

Table 5

b Factors associated with a higher overall quality score of video counseling in a multivariable logistic regression analysis ${ }^{a \star \star}$

\begin{tabular}{|c|c|c|c|}
\hline & $\begin{array}{l}\text { Odds } \\
\text { Ratios }\end{array}$ & $95 \% \mathrm{Cl}$ & $\begin{array}{l}\mathrm{p}- \\
\text { value }\end{array}$ \\
\hline Age (years) & 1.03 & $\begin{array}{l}0.948- \\
1.113\end{array}$ & 0.516 \\
\hline Sex & 0.62 & $\begin{array}{l}0.159- \\
2.444\end{array}$ & 0.498 \\
\hline Referring patients to more blood tests & 0.06 & $\begin{array}{l}0.08- \\
0.378\end{array}$ & 0.003 \\
\hline $\begin{array}{l}\text { Previous experience using the video platforms in primary care } \\
\text { counseling (no experience vs. experience) }\end{array}$ & 1.51 & $\begin{array}{l}0.329- \\
6.956\end{array}$ & 0.59 \\
\hline Constant & 0.84 & & 0.933 \\
\hline \multicolumn{4}{|l|}{${ }^{\text {a }}$ Calculated with the application of sample weights. } \\
\hline
\end{tabular}


In the present study, we described the unique characteristics of consultation during the first phase of the COVID-19 pandemic in Israel. During the outbreak, most primary care physicians reported a decrease in the in-patient visits, while most of them performed mixed counseling by phone, online platforms, or both methods; however, more than half of the respondents implemented telemedicine without previous experience or training in using these modalities. Physicians reported counseling using either phone or online platforms relatively shorter in duration than the usual care.

Telemedicine holds important potential advantages in primary care medicine including continuity of care, monitoring symptoms and behavior in real-time; further, it enables clinicians to reach out to populations who live remotely or are confined to bed $(4,15)$. It has been associated with improved clinical outcomes in various acute conditions such as wound care and chronic diseases such as diabetes mellitus (1) and even follow-up after surgery (16). Telemedicine has even been shown to be as effective as face-to-face care in managing heart failure obtaining glucose control among patients with diabetes (17). During the COVID-19 pandemic, rapid adjustments for delivering telemedicine were reported in several medical fields, for example, neurology (18) and orthopedics (19), while in other fields, such as ophthalmology, only partial solutions were reported (20). Overall, studies reported high satisfaction and high quality of telemedicine. Telephone and video counseling were rated highly by primary care physicians and patients $(3,4)$, with video counseling receiving superior ratings $(21)$. The physicians in our study indicated that delivering care using both telephone and video was reasonable to highly convenient and rated the overall quality of telephone and online platforms relatively high despite pointing it inferior to face-to-face counseling. The majority of the physicians stated they intend to continue using telemedicine options following the crisis.

Despite the substantial benefits of telemedicine, challenges in delivering telemedicine were frequently reported in the literature, including: clinical uncertainty, lack of technological solutions (9), cyber vulnerabilities, and regulation challenges $(8,16)$. In our study, several challenges were highlighted by physicians with regards to their ability to deliver optimal remote care. Physicians reported only moderate technical quality as well as serious organizational difficulties. In qualitative inputs, most physicians reported suboptimal technical quality of the internet or quality of picture or audio. Similar technical problems were reported in other studies $(8,22,23)$. Additional reported barriers were the lack of physical examination and scheduling difficulties (e.g., patients missing appointments or hardship in scheduling appointments).

Remote care poses a challenge regarding resource utilization, as was found in our study. Compared to usual care, telemedicine practice resulted in increases in prescriptions written for antibiotics (56.6\%), blood tests $(16.4 \%)$, referrals to a specialist $(24.5 \%)$, and referrals for imaging $(49.7 \%)$. These findings are consistent with previous research among Israeli pediatricians, in which a $20 \%$ increase in prescription writing for antibiotics for suspected pneumonia or otitis media scenarios was reported (24). Interestingly, the only variable in our study that was significantly associated with a higher reported quality score (both telephone and video) was blood tests - specifically, physicians who did not increase the number of blood tests ordered during the pandemic compared to the usual care reported higher quality scores. Unlike fee- 
per-practices payment systems, the Israeli universal health care system does not grant or deny payment from physicians according to referrals or practices (25). Thus, physicians who avoided submitting additional blood checks may be those with higher confidence in performing the counseling.

In our research, physicians indicated a $40.6 \%$ increase in clinic appointments among younger individuals. Performing counseling to older people, people with hearing disabilities, or lower technological literacy were less satisfactory. Those challenges align with those from a recent review that examined inequalities in the primary care setting. The review concluded that telephone consultations were used more by younger working-age people, the very old, and non-immigrants, with internet-based consultations more likely to be used by younger people (26). An additional survey from Israel reported a positive association between younger age and higher eHealth literacy and satisfaction and usage of telemedicine (27).Efforts should be made to prevent inequalities in delivering primary care due to structural differences. Despite relatively high patient comfort to receive remote care, some prevalent difficulties were reported. Technical difficulties were most prevalent, followed by interpersonal communication difficulties.

Our study emphasizes telemedicine's potential benefits, allowing continuous medical care while ensuring the necessary patient and physician safety in light of possible crisis-induced lockdown. Israeli healthcare and HMOs should address reported challenges; improve technical platforms and scheduling difficulties; and invest in enhancing accessibility of telehealth to older people and patients with technological challenges, thus improving equitable healthcare. Specific training and care modalities should be developed to address interpersonal difficulties and the lack of physical examinations. Israeli healthcare should invest resources to examine alternatives to optimize remote counseling $(28,29)$ through randomized clinical trials and include patient-reported outcomes. Further research is needed to assess the costs and system resource allocation and examine whether usage of medical resources was necessary or preventable. Economic analysis should compare the actual cost and cost-efficacy of extra resource usage reported by our sample in the pandemic to improve telehealth drove medical decision-making models.

The present study has several limitations. First, our data are cross-sectional, and as such, causality cannot be inferred. Second, since we used a convenience sample, and the characteristics of the respondents do not represent the target population, the generalizability of our findings may be limited. Specifically, the study population was younger and included a larger proportion of women than the target population. Despite the lack of representativeness, we have applied sample weights in all our statistical analyses. Our findings were stable and showed consistency between weighted and unweighted samples. We thus conclude that the findings may be generalizable to the target population. It is possible that those who responded to the survey were those who are also more oriented to telemedicine platform usage. However, the unusual circumstances that forced all physicians to respond quickly to the lockdown and the timing of the survey may indicate that these are real-time responses. Our data collection was performed early on during the first phase of COVID pandemic, the healthcare response and the nature of the pandemic have changed since. In a recent survey, conducted among primary care providers and patients, both reported high levels of satisfaction with telemedicine visits in a primary care setting. Both providers and patients reported a desire to see telemedicine visits continued after the pandemic. Benefits 
were highly associated with patients who needed to drive more than 30 minutes to the visit and when the technical quality was high (30). Additional information from a web-based Israeli survey among patients emphasizes that while majority of $80 \%$ patients used telemedicine during the lockdown, men and people with chronic diseases obtained higher willingness to use telemedicine in the future (31). These recent publications, and additional ones (32), indicate that a growing number of individuals in OECD countries are now readily served by telehealth systems (33). It seems that the circumstances have encouraged more providers and patients to use telemedicine as part of their routines. Efforts should be made to improve quality and test modalities of care using this technology.

\section{Conclusions}

Our findings suggest that telemedicine served as a reasonable method for delivering primary care consultations during a global pandemic. However, additional research is needed to establish appropriate standards of care and practice using telemedicine in both crisis and routine periods.

\section{List Of Abbreviations}

COPD, chronic obstructive pulmonary disease; COVID-19, Coronavirus Disease 2019; HMO, Health maintenance organizations; IQR, Intra quartile range; IAFP, Israel Association of Family Physicians

\section{Declarations}

The study was approved by the Institutional Ethics Board (Helsinki Committee) of Ariel University, Israel. Each participant provided a written informed consent prior to responding to the survey. Individuals who did not provide informed consent (indicated by clicking on the appropriate button) could not proceed with the survey. All methods were performed in accordance with the relevant guidelines and regulations by including a statement in the "ethical approval and consent to participate" section to this effect (Declaration of Helsinki).

\section{Consent for publication}

Not applicable

\section{Availability of data and materials}

The datasets generated during and analyzed during the current study are not publicly available due to lack of agreement of the medical organization who distributed the questionnaire. However, data will be available from the corresponding author if a specific reasonable request will be transferred.

\section{Funding}

This research received no funding 


\section{Competing interests}

All authors declare that they have no competing interests. MS, AL, and SV are members of the Israel association of family physicians.

\section{Statement of Authors' contributions}

V.K.S., R.B. and M.S., A.L. and S.V. designed research; V.K.S., R.B., and M.S. conducted research; V.K.S. performed statistical analysis; V.K.S., R.B., M.S. and M.B. wrote the paper. M.S., A.L., and S.V. made significant contributions to data interpretation and development of the discussion. All authors have read and approved the final manuscript.

\section{Acknowledgments}

We are grateful to the study participants for their valuable contribution and to the Israel association of family physicians for their assistance in the distribution of the survey questionnaire.

\section{References}

1. Seehusen DA, Azrak A. The Effectiveness of Outpatient Telehealth Consultations. Am Fam Physician. 2019;100(9):575-7.

2. Orozco-Beltran D, Sánchez-Molla M, Sanchez JJ, Mira JJ. Telemedicine in Primary Care for Patients With Chronic Conditions: The ValCrònic Quasi-Experimental Study. J Med Internet Res. 2017;19(12).

3. Donelan K, Barreto EA, Sossong S, Michael C, Estrada JJ, Cohen AB, et al. Patient and clinician experiences with telehealth for patient follow-up care. Am J Manag Care. 2019;25(1):40-4.

4. Donaghy E, Atherton H, Hammersley V, McNeilly H, Bikker A, Robbins L, et al. Acceptability, benefits, and challenges of video consulting: a qualitative study in primary care. $\mathrm{Br} \mathrm{J}$ Gen Pract. 2019;69(686):e586-e94.

5. Martinez KA, Rood M, Jhangiani N, Kou L, Rose S, Boissy A, et al. Patterns of Use and Correlates of Patient Satisfaction with a Large Nationwide Direct to Consumer Telemedicine Service. J Gen Intern Med. 2018;33(10):1768-73.

6. Siniscalchi M, Zingone F, Savarino E, D'Odorico A, Ciacci C. COVID-19 pandemic perception in adults with celiac disease: an impulse to implement the use of telemedicine. Dig Liver Dis. 2020;52(10):1071-5. doi 10.16/j.dld.2020.05.014. Epub May 16.

7. Peabody MR, Dai M, Turner K, Peterson LE, Mainous AG, 3rd. Prevalence and Factors Associated with Family Physicians Providing E-Visits. J Am Board Fam Med. 2019;32(6):868-75.

8. Scott Kruse C, Karem P, Shifflett K, Vegi L, Ravi K, Brooks M. Evaluating barriers to adopting telemedicine worldwide: A systematic review. J Telemed Telecare. 2018;24(1):4-12.

9. Haimi M, Brammli-Greenberg S, Waisman Y, Baron-Epel O. Physicians' experiences, attitudes and challenges in a Pediatric Telemedicine Service. Pediatr Res. 2018;84(5):650-6. 
10. Gonçalves-Bradley DC, AR JM, Ricci-Cabello I, Villanueva G, Fønhus MS, Glenton C, et al. Mobile technologies to support healthcare provider to healthcare provider communication and management of care. Cochrane Database Syst Rev. 2020;8(8).

11. Barayev E, Shental O, Yaari D, Zloczower E, Shemesh I, Shapiro M, et al. WhatsApp Tele-Medicine usage patterns and physicians views on the platform. Isr J Health Policy Res. 2021;10(1):34. doi 10.1186/s13584-021-00468-8.

12. Vidal-Alaball J, Flores Mateo G, Garcia Domingo JL, Marín Gomez X, Sauch Valmaña G, RuizComellas A, et al. Validation of a Short Questionnaire to Assess Healthcare Professionals' Perceptions of Asynchronous Telemedicine Services: The Catalan Version of the Health Optimum Telemedicine Acceptance Questionnaire. Int J Environ Res Public Health. 2020;17(7).

13. Kaufman-Shriqui V, Sherf-Dagan S, Boaz M, Birk R. Virtual nutrition consultation: what can we learn from the COVID-19 pandemic? Public Health Nutr. 2021;13:1-8.

14. Ginat A. Practitioners and specialists in family medicine in Israel, 2018. Jerusalem, Israel: Administration of Strategic and Economic Planning; 2019.

15. Rauschenberg C, Hirjak D, Ganslandt T, Schulte-Strathaus JCC, Schick A, Meyer-Lindenberg A, et al. [Digital forms of service delivery for personalized crisis resolution and home treatment]. Nervenarzt. 2021;17:1-9.

16. McMaster T, Wright T, Mori K, Stelmach W, To H. Current and future use of telemedicine in surgical clinics during and beyond COVID-19: A narrative review. Ann Med Surg (Lond). 2021;66:102378. doi 10.1016/j.amsu.2021.. Epub 2021 May 8.

17. Flodgren G, Rachas A, Farmer AJ, Inzitari M, Shepperd S. Interactive telemedicine: effects on professional practice and health care outcomes. Cochrane Database Syst Rev. 2015;7(9).

18. Grossman S, Han S, Balcer L, Kurzweil A, Weinberg H, Galetta S, et al. Rapid implementation of virtual neurology in response to the COVID-19 pandemic. Neurology. 2020;94(24):1077-87. doi 10.212/WNL.0000000000009677. Epub 2020 May 1.

19. Tanaka M, Oh L, Martin S, Berkson E. Telemedicine in the Era of COVID-19: The Virtual Orthopaedic Examination. J Bone Joint Surg Am. 2020;102(12):e57. doi 10.2106/JBJS.20.00609.

20. Saleem S, Pasquale L, Sidoti P, Tsai J. Virtual Ophthalmology: Telemedicine in a COVID-19 Era. Am J Ophthalmol. 2020;216:237-42. doi 10.1016/j.ajo.2020.04.029. Epub Apr 30.

21. McConnochie K. Webside Manner: A Key to High-Quality Primary Care Telemedicine for All. Telemed J E Health. 2019;25(11):1007-11. doi 10.89/tmj.2018.0274. Epub 2019 Jan 16.

22. Shah S, Diwan S, Kohan L, Rosenblum D, Gharibo C, Soin A, et al. The Technological Impact of COVID-19 on the Future of Education and Health Care Delivery. Pain Physician. 2020;23(4S):S367S80.

23. Lawrence K, Hanley K, Adams J, Sartori DJ, Greene R, Zabar S. Building Telemedicine Capacity for Trainees During the Novel Coronavirus Outbreak: a Case Study and Lessons Learned. J Gen Intern Med. 2020;35(9):2675-9. 
24. Grossman Z, Chodick G, Reingold S, Chapnick G, Ashkenazi S. The future of telemedicine visits after COVID-19: perceptions of primary care pediatricians. Isr J Health Policy Res. 2020;9(1):53. doi 10.1186/s13584-020-00414-0.

25. Clarfield A, Manor O, Nun G, Shvarts S, Azzam Z, Afek A, et al. Health and health care in Israel: an introduction. Lancet. 2017;389(10088):2503-13. doi 10.1016/S0140-6736(17)30636-0. Epub 2017 May 8.

26. Parker R, Figures E, Paddison C, Matheson J, Blane D, Ford J. Inequalities in general practice remote consultations: A systematic review. BJGP Open. 2021;12(10).

27. Dopelt K, Avni N, Haimov-Sadikov Y, Golan I, Davidovitch N. Telemedicine and eHealth Literacy in the Era of COVID-19: A Cross-Sectional Study in a Peripheral Clinic in Israel. Int J Environ Res Public Health. 2021;18(18):9556. doi 10.3390/ijerph18189556.

28. Temesgen Z, DeSimone D, Mahmood M, Libertin C, Varatharaj PB, Berbari E. Health Care After the COVID-19 Pandemic and the Influence of Telemedicine. Mayo Clin Proc. 2020;95(9S):S66-S8. doi 10.1016/j.mayoc 2020.06.052. Epub Jul 27.

29. Thompson MA, Fuhlbrigge AL, Pearson DW, Saxon DR, Oberst-Walsh LA, Thomas JF. Building eConsult (Electronic Consults) Capability at an Academic Medical Center to Improve Efficiencies in Delivering Specialty Care. J Prim Care Community Health. 2021;12(21501327211005303):21501327211005303.

30. Vosburg R, Robinson K. Telemedicine in Primary Care During the COVID-19 Pandemic: Provider and Patient Satisfaction Examined. Telemed J E Health. 2021;17(10).

31. Reicher S, Sela T, Toren O. Using Telemedicine During the COVID-19 Pandemic: Attitudes of Adult Health Care Consumers in Israel. Front Public Health. 2021;9:653553. doi 10.3389/fpubh.2021.. eCollection 2021.

32. Gamus A, Chodick G. Telemedicine after COVID-19: The Israeli Perspective. Isr Med Assoc J. 2020;22(8):467-9.

33. Wang F, Wang J. The Determinants of Telehealth Provision: Empirical Evidence from OECD Countries. Int J Environ Res Public Health. 2021;18(16):8288. doi 10.3390/ijerph18168288. 